\title{
The risk of venous thromboembolic events in patients with inflammatory bowel disease: a systematic review and meta-analysis
}

\author{
Konstantinos D. Arvanitakis ${ }^{\mathrm{a}, \mathrm{b}}$, Alexandra D. Arvanitaki ${ }^{\mathrm{a}, \mathrm{c}}$, Christos D. Karkos ${ }^{\mathrm{a}, \mathrm{d}}$, Elias A. Zintzaras \\ Georgios S. Germanidis ${ }^{b}$ \\ University of Thessaly, School of Medicine, Larissa, Greece; AHEPA University Hospital, Aristotle University of \\ Thessaloniki, Greece; University Hospital of Muenster, Muenster, Germany; Aristotle University of Thessaloniki, \\ Hippokratio Hospital, Thessaloniki, Greece
}

\section{Abstract}

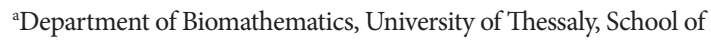
Medicine, Larissa, Greece (Konstantinos D. Arvanitakis, Alexandra D. Arvanitaki, Christos D. Karkos, Elias A. Zintzaras); ${ }^{\text {b }} 1^{\text {st }}$ Department of Internal Medicine, AHEPA University Hospital, Aristotle University of Thessaloniki, Greece (Konstantinos D. Arvanitakis, Georgios S. Germanidis); 'Department of Cardiology III - Center for Adult Congenital and Valvular Heart Disease, University Hospital of Muenster, Muenster,

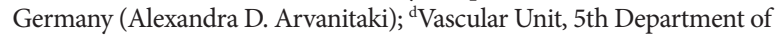
Surgery, Medical School, Aristotle University of Thessaloniki, Hippokratio Hospital, Thessaloniki, Greece (Christos D. Karkos)

Conflict of Interest: Alexandra Arvanitaki is the recipient of the International Training and Research "EMAH Stiftung Karla Voellm" Scholarship, Krefeld, Germany. For the remaining authors none were declared

Correspondence to: Georgios Germanidis, MD, PhD, FEBGH, Associate Professor of Gastroenterology, Gastroenterology Unit, $1^{\text {st }}$ Department of Internal Medicine, AHEPA University Hospital, Aristotle University of Thessaloniki, St. Kiriakidi 1, 54636, Thessaloniki, Greece, e-mail: geogerm@auth.gr

Received 20 December 2020; accepted 18 February 2021; published online 27 May 2021

DOI: https://doi.org/10.20524/aog.2021.0631

\section{Introduction}

Inflammatory bowel disease (IBD) is an autoimmune systemic disorder that mainly affects the gastrointestinal tract and predominantly includes ulcerative colitis (UC) and Crohn's disease $(\mathrm{CD})$, as distinct clinical entities. Its exact cause is not yet fully understood, with genetic susceptibility, environmental factors and alterations in the host's innate as well as adaptive immunity being the principal etiological factors. Apart from the gastrointestinal involvement, IBD is also responsible for a multitude of extraintestinal manifestations, including thromboembolic events (TEs) that significantly increase morbidity and mortality $[1,2]$.

TEs in IBD are often missed, given the fact that the prevalence of thrombosis varies between $1.3 \%$ and $7.7 \%$ in patients with IBD, and the rate increases up to $39-41 \%$ in autopsy series $[3,4]$. The pathologic process of TEs in IBD patients involves multiple factors. Abnormalities in procoagulation, anticoagulation, and fibrinolytic factors have been proven to 
contribute to the development of thrombus in IBD, although several studies have not reported any risk factors in about $50 \%$ of IBD patients with TEs. Acquired risk factors for venous thromboembolism (VTE) include oral contraceptive use, surgical operation, body mass index (BMI) $>30 \mathrm{~kg} / \mathrm{m}^{2}$, trauma, pregnancy, puerperium, lupus anticoagulants, malignancy, long-distance travel, myeloproliferative disorders, and polycythemia vera $[5,6]$. Systemic corticosteroids in long-term courses were also associated with a significantly higher rate of VTE in IBD patients, compared to IBD patients without steroid medication [7]. Over half of the cases of VTE in IBD may be associated with factor $\mathrm{V}$ Leiden and prothrombin gene mutation, which may indicate that genetic factors play a role in VTE; however, this is inconsistent with other studies [8,9].

Venous thrombosis is commonly observed in deep veins of the lower extremities (deep venous thrombosis [DVT]) and the pulmonary arterial circulation (pulmonary embolism $[\mathrm{PE}]$ ). Less frequently, cerebrovascular, portal, mesenteric, hepatic and retinal vein thrombosis is observed [10,11]. Even though several observational studies and a couple of metaanalyses have investigated the relationship between VTE and IBD [12-14], the exact risk estimate of VTE in the IBD population remains ambiguous because of methodological differences and heterogeneity across studies. The aim of this study was to evaluate the risk of VTE in patients with IBD compared to the non-IBD population. Accordingly, we conducted a systematic review and meta-analysis of observational studies that investigated the incidence of VTE, including DVT and/or PE in patients with IBD.

\section{Materials and methods}

This systematic review is reported according to the Preferred Reporting Items for Systematic Reviews and MetaAnalyses (PRISMA) and Meta-analysis Of Observational Studies in Epidemiology (MOOSE) guidelines [15,16]. The study protocol is registered with the PROSPERO international prospective register of systematic reviews (protocol number: CRD42020204404) [17]. The present meta-analysis was performed based on previously published studies; therefore, no ethical approval or patient consent were required.

\section{Search strategy}

A systematic literature search of Medline, Cochrane Library and Scopus was conducted from inception till August 10, 2020, to identify studies that reported the risk of VTE in patients with IBD. Key questions were formulated according to the "PICO" method: "Do adults diagnosed with IBD have increased risk for VTE compared to adults without IBD?" [18]. Text words and, if applicable, database subject heading fields (e.g., Medical Subject Headings), were used to perform the searches: "inflammatory bowel disease", "IBD”, “ulcerative colitis", “Crohn's disease”, "thromboembolism", "pulmonary embolism", “deep vein thrombosis", "thrombosis", "venous thromboembolism", "VTE" and "DVT" (Supplementary material). In addition, we examined the references of each of the retrieved studies to identify further articles that met our criteria. Search filters of human and English language studies were used. The title and abstract of studies identified in the original search were reviewed by 2 independent authors (KA and AA) to eliminate studies that did not answer our research question, based on predetermined inclusion and exclusion criteria. The full text of the remaining articles was evaluated to determine whether it contained pertinent information. The coefficient of agreement between the 2 reviewers for article selection ( $\mathrm{k} 1 / 4$ 0.87, 95\% confidence interval [CI] 0.77-0.96) was excellent. Conflicts in study selection were resolved by consensus, referring back to the original article and, if an agreement failed to be reached, a third author (CK) was consulted.

\section{Selection criteria}

Studies in this meta-analysis were observational cohort or case-control studies that met the following inclusion criteria: 1) diagnosed IBD (CD and/or UC) according to well-defined criteria; 2) reported incident cases of first VTE/ DVT or PE event after the diagnosis of IBD; 3) included a non-IBD population for which VTE/ DVT or PE event rates were calculated (or could be inferred as expected event rates from a reference population); 4) reported relative risk ([RR] for cohort studies), rate or risk ratio (for cohort studies), odds ratios (for case-control studies), hazard ratio (for cohort studies) with $95 \%$ CIs or provided raw data for their calculation; and 5) assessed age and sex as confounding factors. We included peer-reviewed, observational controlled data (case-control and cohort studies) deriving from hospital, referral center and population based-studies. Studies evaluating only pediatric patients (age $<18$ years) were excluded. Cross-sectional studies, meta-analyses, review articles, short surveys, letters to the editor, notes, case reports, pilot studies and conference abstracts were excluded. In addition, studies including only pregnant or postoperative populations as control groups, studies that evaluated only recurrent VTEs and studies that did not contain primary data were excluded. Selection was not restricted by the number of participants in each study. If there were multiple published studies coming from the same population, only data from the most recent comprehensive report were included. VTE was defined as the presence of a first episode of DVT and/or PE, confirmed by objective imaging techniques. IBD, which included UC and/or CD, was defined based on medical diagnostic codes and records of clinical, endoscopic, histological, and radiological findings.

\section{Data extraction}

Two investigators (KA and AA) reviewed and abstracted the data independently onto a standardized form. The following data were collected from the studies: author and year of publication, 
study design, time period of study conduction, origin of the study population, type of exposure (IBD [CD and/or UC] and control population), primary outcome (VTE, DVT, and/or $\mathrm{PE}$ ) and definition of outcome, total number of participants in each group (IBD vs. non-IBD controls), frequency of VTE, DVT, and PE adjusted for potential confounders, as well as confounding factors reported in each study. When frequencies of IBD patients and associated VTE events were not reported in the studies, we merged data on UC and CD to evaluate the VTE risk estimate for the IBD population as a whole. Risk estimates of outcomes were extracted as RRs and their 95\%CIs. Data on the following covariates for DVT or PE were extracted from each study, wherever available: age, sex, history of cancer, history of major surgery, BMI, history of pregnancy, history of PE or DVT, and smoking habits.

\section{Outcome measures}

The primary analysis focused on assessing the RR of VTE, defined as DVT and/or PE, in patients diagnosed with IBD (CD and/or UC) according to well-defined criteria [19-21], compared with non-IBD subjects originating from the general population, hospital or referral center. Furthermore, based on information available from individual studies, we performed subgroup analysis evaluating the risk estimates for DVT and PE separately in patients diagnosed with IBD compared to controls, and additionally compared the risk for DVT vs. PE in IBD patients. Moreover, CD and UC risk estimates for VTE, DVT and PE compared to controls were calculated individually. In addition, risk estimates for VTE, DVT and PE events were separately estimated in patients with UC vs. CD. Risk estimates for VTE in IBD individuals were also calculated, according to the IBD population size. We separated studies into 2 groups (larger and smaller) based on the median value of the total number of IBD patients in each of the 11 studies, which was 13,756 . The influence of sex on the occurrence of VTE in patients with IBD was also assessed. Finally, we assessed the risk for VTE in IBD patients vs. controls adjusted for BMI and smoking, based on the available data.

\section{Data presentation}

The PRISMA flow chart was used to report the selection process of the studies and includes an overall summary of the number and types of articles incorporated into the review (Fig. 1).

\section{Quality assessment of the studies}

The quality of case-control and cohort studies included in our meta-analysis was independently assessed by 2 investigators (KA and AA), using the Newcastle-Ottawa scale [22]. The scale is based on a "star system" that ranges from $0-9$, with 0 being the lowest possible quality, and judges study quality according to 3 perspectives: selection of the study groups (4 questions), comparability of the groups (2 questions), and ascertainment of the outcome of interest (3 questions). Each question was rated with maximum 1 star except for "comparability of the groups", for which separate stars were awarded for controlling age and/or sex (maximum 2 stars). Any differences between the 2 investigators were addressed via a reevaluation of the original article.

\section{Statistical analysis}

All information was reported according to the PRISMA and MOOSE guidelines for meta-analyses $[15,16]$. The Cochrane Collaboration's Review Manager Software (version 5.4) was used to perform the data analysis. The generic inverse variance method was used to combine the studies with different scales of outcome estimates for random-effect meta-analysis, calculating $\ln (\mathrm{RRs})$ and the $\mathrm{SE}(\ln (\mathrm{RR}))$ [23]. When RRs and their respective confidence intervals (CIs) were not reported in the studies, they were calculated using the original data. Because the evaluated outcomes are relatively rare and the effects estimated are generally small, odds ratios in case-control studies were considered reasonable approximations of the corresponding RRs in cohort studies $[24,25]$. We assessed the heterogeneity between study-specific estimates using 2 methods. First, the Cochran Q statistical test for heterogeneity, which tests the null hypothesis that all studies in a meta-analysis have the same underlying magnitude of effect, was measured. Because this test is underpowered to detect moderate degrees of heterogeneity, the presence of statistically significant heterogeneity across the studies was evaluated by utilizing a $\mathrm{P}$-value $<0.10$. Second, to estimate what proportion of total variation across studies was caused by study-related factors (clinical setting, methodological or statistical differences) rather than chance, the $\mathrm{I}^{2}$ statistic was calculated, where $I^{2}=100 \% \times(\mathrm{Q}-\mathrm{df}) / \mathrm{Q}$ represents the magnitude of the heterogeneity (moderate: 30-60\%, substantial: 50-90\%, considerable: $75-100 \%$ ) [23,26,27]. Dichotomous outcomes were pooled using the Mantel-Haenszel random-effects model (when more than moderate heterogeneity was detected among studies), used to calculate the RRs and corresponding 95\%CIs. The Mantel-Haenszel fixed effects model was used to calculate the RRs and corresponding 95\%CIs in the case of homogenous studies. For all tests (except for heterogeneity), a probability level $<0.05$ was considered statistically significant.

Visual inspection of the funnel plot demonstrated the asymmetry typically associated with publication bias [28]. That is, smaller, less precise studies (those with the larger standard errors) appeared to have higher RRs than the larger, more precise studies. Evidence of publication bias was also confirmed by Egger's test [29], which was performed using linear regression analysis in the IBM SPSS statistics 26.0 software, since the number of the included studies was $\mathrm{n}>10$. 


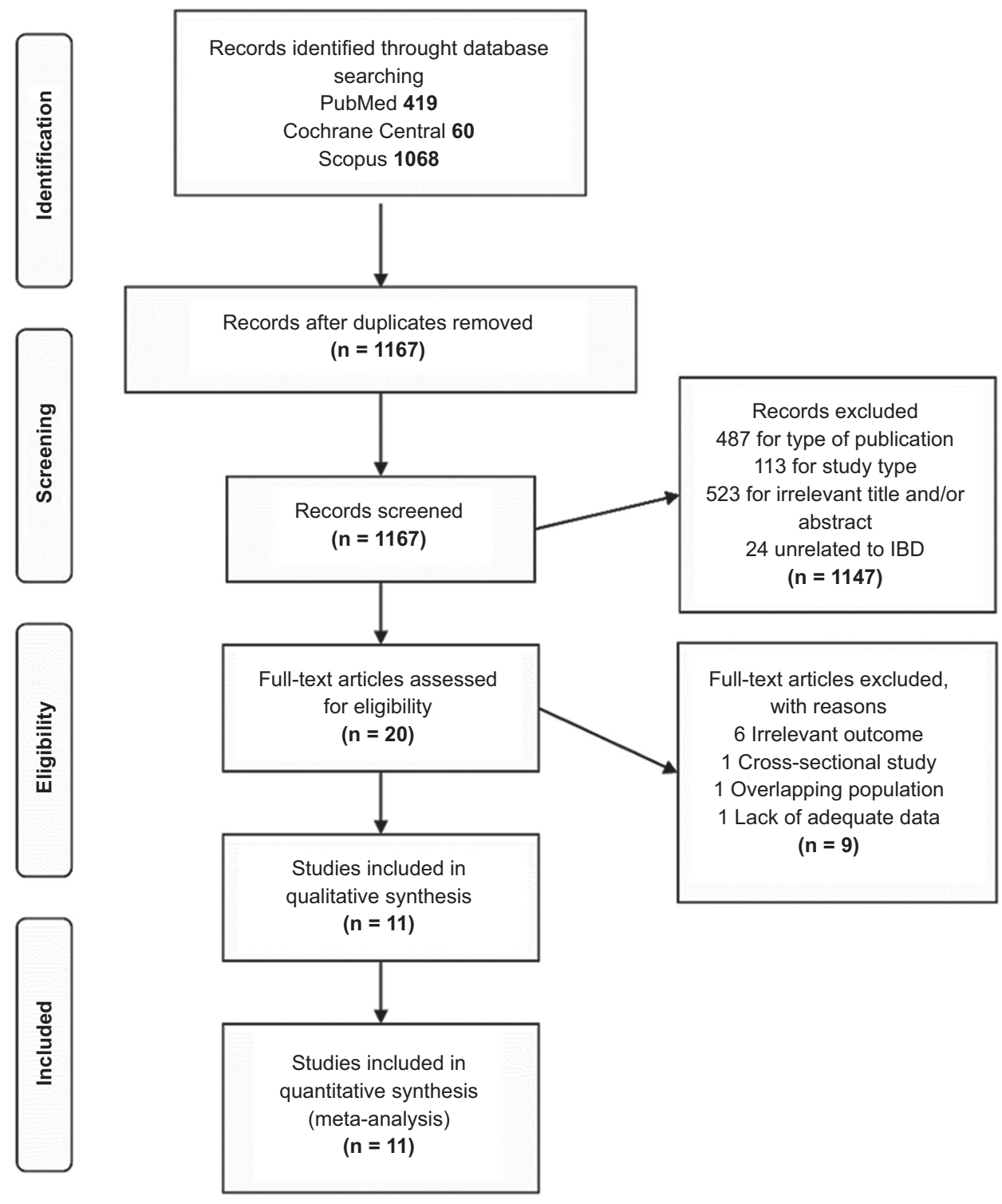

Figure 1 Study selection flow diagram presented according to the PRISMA Statement

\section{Results}

\section{Eligible studies}

The search strategy identified 1547 articles (Fig. 1). After removal of duplicates and screening of titles, abstracts and keywords, 20 papers underwent full-text review. During this process, 6 articles were excluded because of irrelevant outcomes [30-35], while 1 study was excluded for its crosssectional design [36]. In addition, 2 studies reported outcomes that originated from the same database [37,38]; accordingly, the study with the shorter follow-up period was excluded [38]. Another study was excluded because it neither provided an overall risk estimate for VTE, nor offered available data for calculating it [39]. The remaining 11 studies [37,40-49], published between 2001 and 2018, fulfilled the selection criteria.

\section{Quality assessment of the included studies}

We used the Newcastle-Ottawa scale to evaluate the quality of the studies included in our meta-analysis: 2 studies were rated as 9-star, 5 studies as 8 -star and 4 studies as 7 -star. The included studies averaged a quality score of 7.8. All studies provided a clear definition of the diagnosis of VTE, including the details of confirmation based on imaging techniques. Some studies used the international disease codes for VTE diagnosis (Table 1). 
Table 1 The Newcastle-Ottawa scale for assessing the risk of bias of the included studies

\begin{tabular}{|c|c|c|c|c|}
\hline Study, year [Ref.] & $\begin{array}{c}\text { Selection } \\
\text { (max } 4 \text { stars) }\end{array}$ & $\begin{array}{l}\text { Comparability } \\
\text { (max } 2 \text { stars) }\end{array}$ & $\begin{array}{l}\text { Exposure/outcome } \\
\quad(\max 3 \text { stars })\end{array}$ & $\begin{array}{l}\text { Overall quality score } \\
\quad(\max 9 \text { stars })\end{array}$ \\
\hline Нa 2004 [44] & $* * * *$ & * & $* *$ & 7 \\
\hline Kappelman 2011 [37] & $* * * *$ & * & $* * *$ & 8 \\
\hline Bernstein 2001 [40] & $* * * *$ & * & $* * *$ & 8 \\
\hline Chung 2015 [42] & $* * * *$ & * & $* * *$ & 8 \\
\hline Grainge 2010 [43] & $* * * *$ & $* *$ & $* * *$ & 9 \\
\hline Miehsler 2004 [45] & $* * *$ & ** & $* *$ & 7 \\
\hline Nguyen 2008 [46] & $* * * *$ & * & $* *$ & 7 \\
\hline Rothberg 2011 [47] & $* * * *$ & * & $* *$ & 7 \\
\hline Saleh 2010 [48] & $* * * *$ & * & $* *$ & 8 \\
\hline Weng 2018 [49] & $* * * *$ & * & $* * *$ & 8 \\
\hline Chu 2018 [41] & $* * * *$ & $* *$ & $* * *$ & 9 \\
\hline
\end{tabular}

A "star" symbol identifies "high" quality choices. The score allows a maximum of 1 "star" for each item within the "Selection" and "Outcome" categories and a maximum of 2 "stars" for "Comparability". Highest quality choice allows 4 stars for "Selection", 2 stars for "Comparability" and 3 stars for "Outcome". In comparability, 1 star was allocated in studies that adjusted for the confounder "age" and 2 stars in studies that also adjusted for the confounder "smoking"

\section{General characteristics}

Our meta-analysis included 11 observational studies (10 cohort studies [40-49] and 1 case-control study [37]), the general characteristics of which are described in Table 2. Six studies were population-based, 4 were hospital-based and 1 study had a referral center as a population source. The primary analysis included $3,175,012$ patients with IBD and $920,144,253$ controls without IBD. In the analysis of secondary outcomes, regarding the evaluation of risk estimates for DVT and PE in IBD patients compared to controls without IBD, 5 studies were included $[37,42,44,45,49]$. In addition, 4 studies were included in the analysis regarding the risk of VTE in patients with UC compared to patients with CD $[37,44,48,49]$, while 3 studies were used to evaluate the risk of DVT and PE $[37,44,49]$, in the same subgroups. Articles had large differences in their selection of covariates for adjusted analyses. Covariates included demographics such as age and sex, past medical history (history of surgery, history of pregnancy, history of cancer, history of PE or DVT), as well as other risk factors, such as BMI and smoking habits. Six studies included in the analysis assessed the influence of sex on the risk for VTE in patients with IBD $[37,41,42,45,48,49]$. A subgroup analysis was also performed, including 3 studies that evaluated smoking and BMI as confounding factors $[41,43,45]$.

\section{Quantitative analysis and evaluation of heterogeneity}

The overall RR for VTE in patients with IBD compared to non-IBD individuals was 2.03 (95\%CI 1.72-2.39). All individual studies had RR estimates above 1.0 with statistical significance. Significant heterogeneity was observed among studies (Q statistic=412.43, $\mathrm{P}<0.10, I^{2}=98 \%$ ). (Fig. 2).
To identify possible sources of heterogeneity, several subgroup analyses were performed relating to study size, IBD type, thrombosis location, and adjustment for confounders (Table 3). Analysis of studies with larger IBD population size $[37,41,43,44,46,48]$ demonstrated a lower risk for VTE (RR 1.77, 95\%CI 1.48-2.13) among patients with IBD, whereas studies with smaller IBD population size $[40,42,45,47,49]$ yielded a greater risk for VTE (RR 2.67, 95\%CI 1.97-2.93). A greater risk for VTE was found in both patients with UC (RR 1.8, 95\%CI 1.15-2.82) and those with CD (RR 1.72, 95\%CI 1.58-1.88), with no difference between the 2 groups (RR 1.03, 95\%CI 0.72-1.46). Similar results were found in relation to the risk for DVT and $\mathrm{PE}$ in patients with UC and CD, with no difference between the 2 IBD clinical entities (Table 3). Additionally, patients with IBD presented a greater risk for DVT (RR 1.95, 95\%CI 1.592.39) and PE (RR 1.91, 95\%CI 1.75-2.08) compared to controls. The risk for DVT was almost 2-fold higher than that for PE in the IBD population (RR 1.96, 95\%CI 1.34-2.86). Moreover, the risk for VTE in patients with IBD did not differ statistically significantly between male and female patients (RR 0.95, 95\%CI 0.8-1.12). After adjustment for smoking and BMI, the RR for VTE was moderately increased (RR 2.65, 95\%CI 1.51-4.65). The remaining 8 studies that did not adjust for these confounders demonstrated a lower risk for VTE in patients with IBD, but still greater compared to controls (RR 1.88, 95\%CI 1.57-2.24).

There was significant heterogeneity among all the studies included in the aforementioned subgroup analyses (Table 3). Only studies evaluating the risk for PE in patients with IBD compared to non-IBD subjects demonstrated no evidence of heterogeneity $\left(\chi^{2}=2.06, \mathrm{P}=0.72, I^{2}=0 \%\right)$. Finally, a secondary analysis was performed using the most recently published studies $[41,42,49]$, which demonstrated a higher risk of VTE in patients with IBD (RR 1.88, 95\%CI 1.70-2.07), without evidence of heterogeneity among them ( $Q$ statistic $=0.43$, $\mathrm{P}=0.81 I^{2}=0 \%$ ) (Table 3). 
6 K. D. Arvanitakis et al

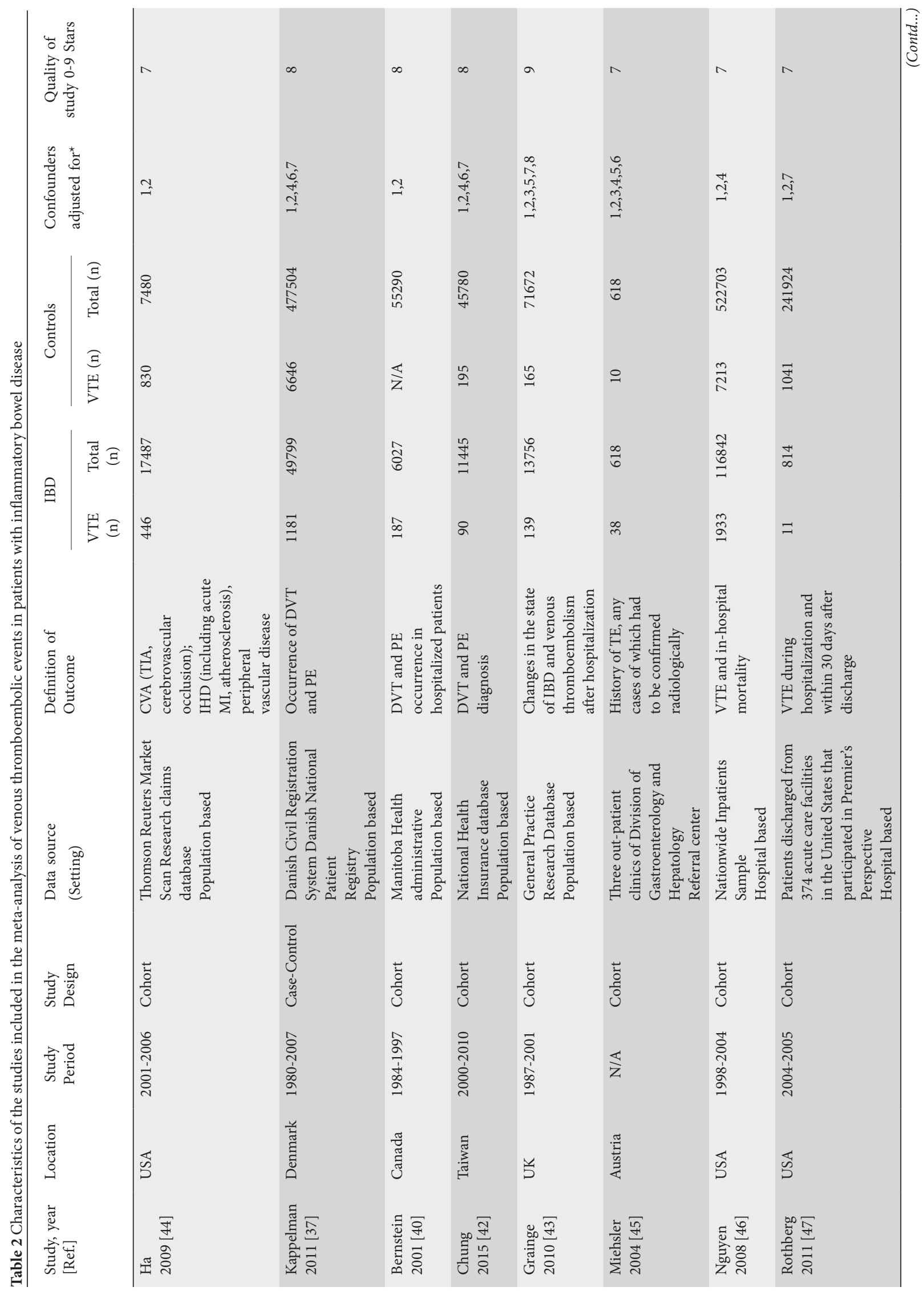




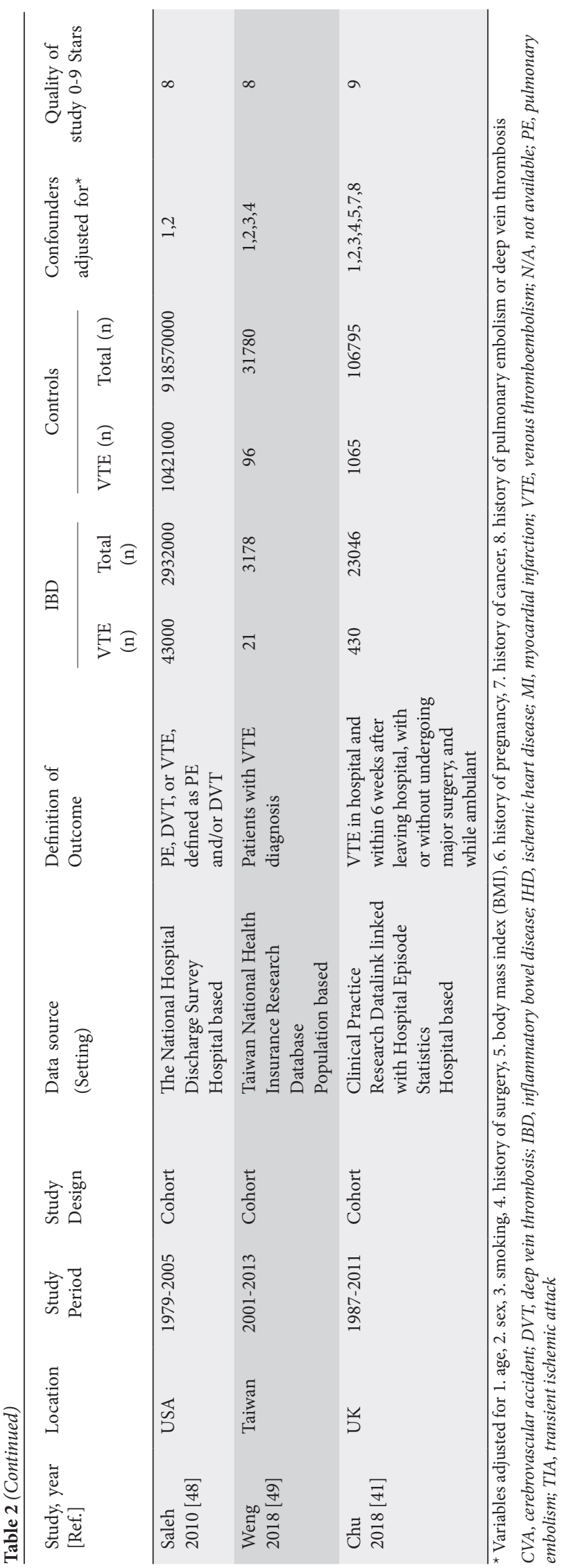

\section{Publication bias}

Upon inspection, the funnel plot of studies included in the primary analysis showed evidence of asymmetry suggestive of publication bias, which was confirmed statistically (Egger $\mathrm{P}=0.017$ ) (Fig. 3). After 6 studies that appeared to be the cause of the asymmetry $[40,43,44,46-48]$ were removed, little to no evidence of underlying bias could be found. Similarly, visual inspection of the funnel plots of the studies included in most of the subgroup analyses presented asymmetry that was indicative of possible publication bias.

\section{Discussion}

Our meta-analysis, which evaluated pooled data from all currently available observational studies assessing the risk of VTE in patients with IBD, indicated that the overall risk of VTE was 2-fold higher in the IBD group, compared to the non-IBD control group. After both qualitative and quantitative confirmation of funnel plot asymmetry, publication bias was suspected of being the main culprit behind this relationship between VTE and IBD. As the pooled RR for VTE derived from the 6 studies with the largest sample sizes was substantially higher than the pooled RR from the "smaller" ones, the aforementioned assumption seemed quite plausible. However, the pooled RR for VTE derived from the "larger" studies did not differ greatly from the pooled RR of the primary analysis, indicating that publication bias on its own could not have been the causal factor for our results.

Subgroup analysis revealed that the risk of VTE in IBD was moderately increased after adjusting for smoking and BMI, indicating that, when considered together, these factors could act as confounders in the relationship between IBD and VTE. In addition, patients with IBD demonstrated an almost 2-fold higher risk for DVT compared to the risk for PE, in accordance with the yearly incidence of DVT and PE in the general population in Europe and the United States [50,51]. Various studies suggest that there is a $50 \%$ chance for patients with untreated proximal DVT to develop symptomatic PE within 3 months, and that in patients with superficial vein thrombosis, concomitant DVT and PE are prevalent in $18.1 \%$ and $6.9 \%$ of them, respectively [52,53]. Furthermore, the risk for VTE in patients with IBD was not influenced by sex, despite several reports demonstrating a higher risk in men [54,55]. Finally, a greater risk for VTE, as well as for DVT and PE, was found in patients with UC and CD, similar to that of the total IBD population.

In total, 2 meta-analyses have been published previously regarding the risk of VTE in patients with IBD. Fumery et al described an elevated risk of VTE in patients with IBD (RR 1.96, 95\%CI 1.67-2.30) [12]. Yuhara et al, on the other hand, described an even greater risk of VTE in IBD patients (RR $2.20,95 \%$ CI 1.83-2.65) [14]. The slight discrepancy in the size estimates between the previous meta-analyses and this study lies in the fact that different inclusion and exclusion criteria 
Table 3 Results of meta-analyses by type of outcome

\begin{tabular}{|c|c|c|c|c|c|c|}
\hline \multirow[t]{2}{*}{ Outcome of interest } & \multirow[t]{2}{*}{ Number of studies } & \multirow[t]{2}{*}{ RR $(95 \% \mathrm{CI})$} & \multirow[t]{2}{*}{ Effect Model } & \multicolumn{3}{|c|}{ Heterogeneity } \\
\hline & & & & $I^{2}$ & P-value & Q-statistic \\
\hline VTE in IBD & 11 & $2.03(1.72-2.39)$ & Random & $98 \%$ & $<0.01$ & 412.43 \\
\hline DVT in IBD & 5 & $1.95(1.59-2.39)$ & Random & $75 \%$ & $<0.01$ & 16.07 \\
\hline $\mathrm{PE}$ in IBD & 5 & $1.91(1.75-2.08)$ & Fixed & $0 \%$ & 0.72 & 2.06 \\
\hline DVT vs. PE in IBD & 5 & $1.96(1.34-2.86)$ & Random & $86 \%$ & $<0.01$ & 29.23 \\
\hline VTE in UC & 4 & $1.72(1.58-1.88)$ & Random & $74 \%$ & $<0.01$ & 11.54 \\
\hline VTE in CD & 4 & $1.80(1.15-2.82)$ & Random & $98 \%$ & $<0.01$ & 181.37 \\
\hline DVT in CD vs. UC & 3 & $1.06(0.78-1.42)$ & Random & $67 \%$ & 0.05 & 6.13 \\
\hline $\mathrm{PE}$ in $\mathrm{CD}$ vs. $\mathrm{UC}$ & 3 & $1.15(0.89-1.49)$ & Random & $30 \%$ & 0.24 & 2.88 \\
\hline VTE in larger size studies & 6 & $1.77(1.48-2.13)$ & Random & $98 \%$ & $<0.01$ & 319.15 \\
\hline VTE in smaller size studies & 5 & $2.67(1.93-3.71)$ & Random & $72 \%$ & $<0.01$ & 14.39 \\
\hline Male vs. Female VTE in IBD & 6 & $0.95(0.8-1.12)$ & Random & $81 \%$ & $<0.01$ & 25.76 \\
\hline Adjusted VTE risk* & 3 & $2.65(1.51-4.65)$ & Random & $93 \%$ & $<0.01$ & 28.27 \\
\hline Unadjusted VTE risk* & 8 & $1.88(1.57-2.24)$ & Random & $98 \%$ & $<0.01$ & 316.11 \\
\hline
\end{tabular}

${ }^{*}$ Variables adjusted for were smoking and body mass index

IBD, inflammatory bowel disease; CD, Crohn's disease; DVT, deep vein thrombosis; PE, pulmonary embolism; UC, ulcerative colitis; VTE, venous thromboembolism; $R R$, relative risk; CI, confidence interval

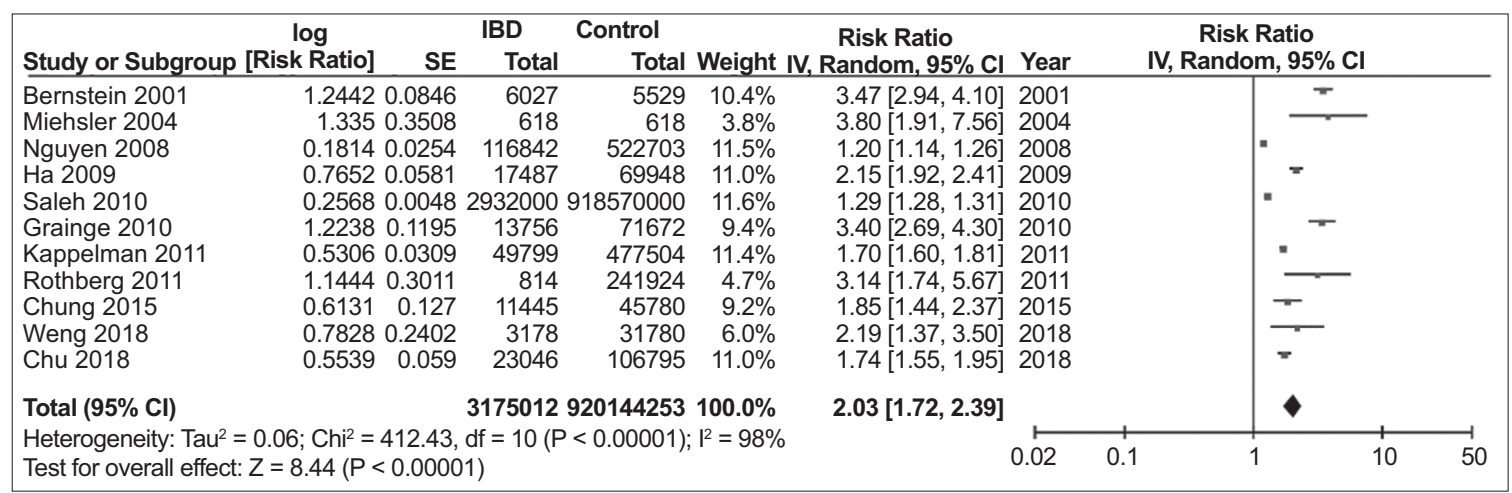

Figure 2 Forest plot demonstrating the summary relative risk for venous thromboembolic events in patients with inflammatory bowel disease. Studies are listed in chronological order.

$I B D$, inflammatory bowel disease; CI, confidence interval

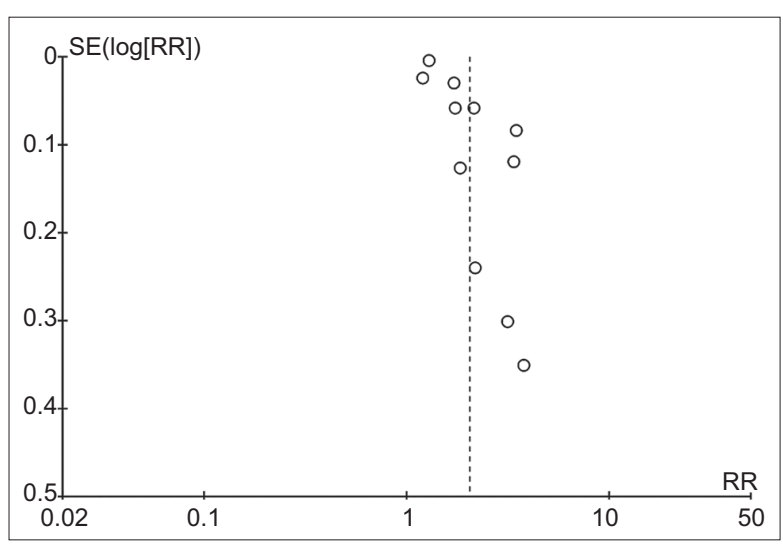

Figure 3 Funnel plot of the 11 studies included in the meta-analysis $S E$, standard error; $R R$, relative risk were used. In contrast to the previously published studies, we specifically excluded articles that included solely postoperative patients or pregnant women with IBD, to avoid selection bias. In general, pregnant women develop VTE 4-5 times more frequently than non-pregnant women [56,57], while a recent meta-analysis estimated the VTE risk in pregnant women with IBD to be 10-fold higher than that of non-pregnant ones without IBD [13]. Furthermore, major surgery has been proven to be a strong risk factor for VTE [58]. In addition, we excluded a study by Bernstein et al, included in the other meta-analyses [39], because it did not satisfy our inclusion criteria; in particular, the frequencies of VTE events in both the IBD population and the controls were not reported in the original study, while there was no overall risk estimate of VTE in patients with IBD; only ageand sex-specific comorbidity rates were reported. 
There is a long-standing debate about the prevention methods and treatment options of VTE in patients with IBD. According to recent consensus statements regarding the prevention and treatment of VTE in patients with IBD [59], moderate to severe disease activity increases the risk of VTE and thus should be considered as a provoking factor. It is alarming that up to date, inadequate use of anticoagulants for VTE prophylaxis in IBD has been reported [60,61], and is mainly attributed to: (1) gastroenterologists' lack of awareness of both the increased risk of VTE in IBD patients and the recommended use of pharmacological prophylaxis in hospitalized IBD patients [62]; and (2) concerns about the safety of anticoagulant drugs in patients with active IBD [63].

Pathophysiologic mechanisms that could explain the increased risk of thromboembolism in IBD have not yet been fully elucidated. Several studies have shown that glucocorticoid use in the setting of increased inflammatory activity, such as in patients with IBD, increases clotting factor levels and levels of plasminogen activator inhibitor-1, suggesting that glucocorticoid-induced alterations in fibrinolysis may contribute to the presence of a hypercoagulable state [64,65]. Moreover, emerging data indicate that neutrophil extracellular traps (NETs) expressing active tissue factor [66] are crucially implicated in the pathogenesis of various thromboinflammatory disorders [67-69]. A recent study pointed out that the local inflammatory response in the colon and peripheral blood in active UC was characterized by the presence of NETs carrying bioactive interleukin- $1 \mathrm{~b}$ and thrombogenic tissue factor, which could be responsible for the higher frequency of thrombosis in those patients than in healthy individuals [70]. Clinical studies have revealed an increased platelet count (reactive thrombocytosis) in IBD patients, proposed as a biomarker of disease activity in human IBD [71]. In addition, thrombocytosis appears to be accompanied by the presence of immature platelets in blood, suggesting that this response to IBD is linked to accelerated thrombopoiesis [72,73]. The intrinsic coagulation pathway can be activated by NETs released by polymorphonuclear neutrophils (PMNs) in a process called NETosis. NETs activate platelets as well as the complement system $(\mathrm{C} 3 \mathrm{a}, \mathrm{C} 5 \mathrm{a})$ and release proteases that inactivate endogenous anticoagulants. Platelets secrete alpha granules that recruit PMNs and macrophages, while stimulating PMNs to undergo NETosis, which in turn reactivates platelets, creating a feedback loop (platelet-mediated NET-driven thrombogenicity). They also activate the coagulation pathway by assembling enzyme-cofactor-substrate complexes on their exposed surface [74-76]. Clinical evaluation and interpretation of these findings could be promising, in order to develop novel diagnostic and therapeutic targets for clinically active IBD patients with an increased risk of immunothrombosis.

Several limitations of this study must be acknowledged. First of all, since no randomized controlled trials have been performed to explore the association between VTE and IBD, our meta-analysis included only observational studies, which are often susceptible to selection bias and may fail to take into account several potential confounders for the risk factor under investigation [77]. Second, significant heterogeneity (>90\%) was observed among studies. That could be attributed to a variety of factors, such as different population characteristics of the included studies: 1) some studies were population-based cohorts, while in others data were abstracted from hospitalized patients or referral centers; and 2) studies had different followup periods and variability in the disease phenotype of IBD among patients. Although IBD severity, colonic involvement and extent of disease have been proven to correlate with VTE risk $[45,46]$, not enough studies provided sufficient information on disease location or disease characteristics and severity, so we were not able to further analyze these factors. Third, since VTE is frequently diagnosed post mortem [78], some degree of differential misclassification of outcomes is to be expected in the studies of this meta-analysis. Finally, we cannot rule out that we omitted relevant articles by having imposed the English language as a filter on our search.

To conclude, patients with IBD carry a 2 -fold greater risk of VTE compared to non-IBD subjects; it is therefore of utmost importance to increase gastroenterologists' awareness of the primary prevention of VTE in this group of patients. Further, adequately conducted prospective cohort studies as well as randomized trials are warranted to provide more robust data regarding risk differences among certain subgroups.

\section{Summary Box}

\section{What is already known:}

- Inflammatory bowel disease (IBD) is responsible for a multitude of extraintestinal manifestations, including thromboembolic events that significantly increase morbidity and mortality

- Thromboembolic events in patients with IBD are often missed, given the fact that the prevalence of thrombosis varies between $1.3 \%$ and $7.7 \%$ in patients with IBD, and the rate rises to $39-41 \%$ in autopsy series

- The exact risk estimate of venous thromboembolism (VTE) in the IBD population still remains ambiguous, because of methodological differences and heterogeneity across published studies

\section{What the new findings are:}

- The overall risk of VTE was found to be 2.03 times higher in the IBD group compared to the non-IBD control group

- The risk of VTE in IBD was moderately increased after adjusting for smoking and body mass index, indicating that, when considered together, these factors could act as confounders in the relationship between IBD and VTE

- Patients with IBD demonstrated an almost 2-fold higher risk for deep venous thrombosis compared to the risk for pulmonary embolism 


\section{References}

1. Danese S, Papa A, Saibeni S, Repici A, Malesci A, Vecchi M. Inflammation and coagulation in inflammatory bowel disease: The clot thickens. Am J Gastroenterol 2007;102:174-186.

2. Murthy SK, Nguyen GC. Venous thromboembolism in inflammatory bowel disease: an epidemiological review. Am J Gastroenterol 2011;106:713-718.

3. Loftus EV Jr. Clinical epidemiology of inflammatory bowel disease: incidence, prevalence, and environmental influences. Gastroenterology 2004;126:1504-1517.

4. Talbot RW, Heppell J, Dozois RR, Beart RW Jr. Vascular complications of inflammatory bowel disease. Mayo Clin Proc 1986;61:140-145.

5. Koutroubakis IE. Therapy insight: Vascular complications in patients with inflammatory bowel disease. Nat Clin Pract Gastroenterol Hepatol 2005;2:266-272.

6. Owczarek D, Cibor D, Głowacki MK, Rodacki T, Mach T. Inflammatory bowel disease: epidemiology, pathology and risk factors for hypercoagulability. World J Gastroenterol 2014;20:53-63.

7. Sarlos P, Szemes K, Hegyi P, et al. Steroid but not biological therapy elevates the risk of venous thromboembolic events in inflammatory bowel disease: a meta-analysis. J Crohns Colitis 2018;12:489-498.

8. Spina L, Saibeni S, Battaglioli T, Peyvandi F, de Franchis R, Vecchi M. Thrombosis in inflammatory bowel diseases: role of inherited thrombophilia. Am J Gastroenterol 2005;100:2036-2041.

9. Yan SL, Russell J, Harris NR, Senchenkova EY, Yildirim A, Granger DN. Platelet abnormalities during colonic inflammation. Inflamm Bowel Dis 2013;19:1245-1253.

10. Algahtani FH, Farag YM, Aljebreen AM, et al. Thromboembolic events in patients with inflammatory bowel disease. Saudi $J$ Gastroenterol 2016;22:423-427.

11. Twig G,Zandman-Goddard G, Szyper-KravitzM, Shoenfeld Y. Systemic thromboembolism in inflammatory bowel disease: mechanisms and clinical applications. Ann N Y Acad Sci 2005;1051:166-173.

12. Fumery M, Xiaocang C, Dauchet L, Gower-Rousseau C, Peyrin-Biroulet L, Colombel JF. Thromboembolic events and cardiovascular mortality in inflammatory bowel diseases: a metaanalysis of observational studies. J Crohns Colitis 2014;8:469-479.

13. Kim YH, Pfaller B, Marson A, Yim HW, Huang V, Ito S. The risk of venous thromboembolism in women with inflammatory bowel disease during pregnancy and the postpartum period: A systematic review and meta-analysis. Medicine (Baltimore) 2019;98:e17309.

14. Yuhara H, Steinmaus C, Corley D, et al. Meta-analysis: the risk of venous thromboembolism in patients with inflammatory bowel disease. Aliment Pharmacol Ther 2013;37:953-962.

15. Moher D, Liberati A, Tetzlaff J, Altman DG; PRISMA Group. Preferred reporting items for systematic reviews and metaanalyses: the PRISMA statement. PLoS Med 2009;6:e1000097.

16. Stroup DF, Berlin JA, Morton SC, et al. Meta-analysis of observational studies in epidemiology: a proposal for reporting. Meta-analysis Of Observational Studies in Epidemiology (MOOSE) group. JAMA 2000;283:2008-2012.

17. Arvanitakis K, Arvanitaki A, Karkos C. The risk of venous thromboembolic events in patients with inflammatory bowel disease: A systematic review and meta-analysis. PROSPERO 2020 CRD42020204404. Available from: https://www.crd.york.ac.uk/ prospero/display_record.php?ID=CRD42020204404 [Accessed 4 April 2021].

18. Fineout-Overholt E, Johnston L. Teaching EBP: asking searchable, answerable clinical questions. Worldviews Evid Based Nurs 2005;2:157-160.

19. Annese V, Daperno M, Rutter MD, et al; European Crohn's and Colitis Organisation. European evidence based consensus for endoscopy in inflammatory bowel disease. J Crohns Colitis 2013;7:982-1018.
20. Dignass A, Van Assche G, Lindsay JO, et al; European Crohn's and Colitis Organisation (ECCO). The second European evidencebased Consensus on the diagnosis and management of Crohn's disease: Current management. J Crohns Colitis 2010;4:28-62.

21. Magro F, Langner C, Driessen A, et al; European Crohn's and Colitis Organisation (ECCO). European consensus on the histopathology of inflammatory bowel disease. J Crohns Colitis 2013;7:827-851.

22. Wells GA, Shea B, O'Connell D, Peterson J, Welch V, Losos M, et al. The Newcastle-Ottawa scale (NOS) for assessing the quality of nonrandomised studies in meta-analyses. 2019. Available from: http://www.ohri.ca/programs/clinical_epidemiology/oxford.asp [Accessed 4 April 2021].

23. Altman DG. Confidence intervals for the number needed to treat. BMJ 1998;317:1309-1312.

24. Nurminen M. To use or not to use the odds ratio in epidemiologic analyses? Eur J Epidemiol 1995;11:365-371.

25. Stare J, Maucort-Boulch D. Odds ratio, hazard ratio and relative risk. Metodološki zvezki 2016;13:59-67.

26. Cochran WG. The combination of estimates from different experiments. Biometrics 1954;10:101-129.

27. Higgins JP, Thompson SG, Deeks JJ, Altman DG. Measuring inconsistency in meta-analyses. BMJ 2003;327:557-560.

28. Higgins JPT TJ, Chandler J, Cumpston M, Li T, Page MJ, Welch VA (editors). Cochrane Handbook for Systematic Reviews of Interventions. 2nd ed. Chichester (UK): John Wiley \& Sons; 2019.

29. Egger M, Davey Smith G, Schneider M, Minder C. Bias in metaanalysis detected by a simple, graphical test. BMJ 1997;315:629-634.

30. Bhandari S, Saeian K, Stein D. Increased rate of venous thromboembolism in hospitalized inflammatory bowel disease patients with Clostridium difficile infection. Inflamm Bowel Dis 2018;24:661.

31. Grip O, Svensson PJ, Lindgren S. Inflammatory bowel disease promotes venous thrombosis earlier in life. Scand J Gastroenterol 2000;35:619-623.

32. Merrill A, Millham F. Increased risk of postoperative deep vein thrombosis and pulmonary embolism in patients with inflammatory bowel disease: a study of National Surgical Quality Improvement Program patients. Arch Surg 2012;147:120-124.

33. Nguyen GC, Boudreau H, Harris ML, Maxwell CV. Outcomes of obstetric hospitalizations among women with inflammatory bowel disease in the United States. Clin Gastroenterol Hepatol 2009;7:329-334.

34. Yarur AJ, Deshpande AR, Pechman DM, Tamariz L, Abreu MT, Sussman DA. Inflammatory bowel disease is associated with an increased incidence of cardiovascular events. Am J Gastroenterol 2011;106:741-747.

35. Zöller B, Ohlsson H, Waehrens R, Sundquist J, Sundquist K. Association of irritable bowel syndrome and venous thromboembolism. Scand J Gastroenterol 2018;53:784-789.

36. Kuy S, Dua A, Chappidi R, et al. The increasing incidence of thromboembolic events among hospitalized patients with inflammatory bowel disease. Vascular 2015;23:260-264.

37. Kappelman MD, Horvath-Puho E, Sandler RS, et al. Thromboembolic risk among Danish children and adults with inflammatory bowel diseases: a population-based nationwide study. Gut 2011;60:937-943.

38. Huerta C, Johansson S, Wallander MA, García Rodríguez LA. Risk factors and short-term mortality of venous thromboembolism diagnosed in the primary care setting in the United Kingdom. Arch Intern Med 2007; 167:935-943.

39. Bernstein CN, Nabalamba A. Hospitalization-based major comorbidity of inflammatory bowel disease in Canada. Can J Gastroenterol 2007;21:507-511.

40. Bernstein CN, Blanchard JF, Houston DS, Wajda A. The incidence of deep venous thrombosis and pulmonary embolism among patients with inflammatory bowel disease: a population-based cohort study. Thromb Haemost 2001;85:430-434.

41. Chu TPC, Grainge MJ, Card TR. The risk of venous thromboembolism during and after hospitalisation in patients with inflammatory bowel 
disease activity. Aliment Pharmacol Ther 2018;48:1099-1108.

42. Chung WS, Lin CL, Hsu WH, Kao CH. Inflammatory bowel disease increases the risks of deep vein thrombosis and pulmonary embolism in the hospitalized patients: a nationwide cohort study. Thromb Res 2015;135:492-496.

43. Grainge MJ, West J, Card TR. Venous thromboembolism during active disease and remission in inflammatory bowel disease: a cohort study. Lancet 2010;375:657-663.

44. Ha C, Magowan S, Accortt NA, Chen J, Stone CD. Risk of arterial thrombotic events in inflammatory bowel disease. Am J Gastroenterol 2009;104:1445-1451.

45. Miehsler W, Reinisch W, Valic E, et al. Is inflammatory bowel disease an independent and disease specific risk factor for thromboembolism? Gut 2004;53:542-548.

46. Nguyen GC, Sam J. Rising prevalence of venous thromboembolism and its impact on mortality among hospitalized inflammatory bowel disease patients. Am J Gastroenterol 2008;103:2272-2280.

47. Rothberg MB, Lindenauer PK, Lahti M, Pekow PS, Selker HP. Risk factor model to predict venous thromboembolism in hospitalized medical patients. J Hosp Med 2011;6:202-209.

48. Saleh T, Matta F, Yaekoub AY, Danescu S, Stein PD. Risk of venous thromboembolism with inflammatory bowel disease. Clin Appl Thromb Hemost 2011;17:254-258.

49. Weng MT, Park SH, Matsuoka K, et al. Incidence and risk factor analysis of thromboembolic events in East Asian patients with inflammatory bowel disease, a multinational collaborative study. Inflamm Bowel Dis 2018;24:1791-1800.

50. Cohen AT, Agnelli G, Anderson FA, et al; VTE Impact Assessment Group in Europe (VITAE). Venous thromboembolism (VTE) in Europe. The number of VTE events and associated morbidity and mortality. Thromb Haemost 2007;98:756-764.

51. Anderson FA Jr, Zayaruzny M, Heit JA, Fidan D, Cohen AT. Estimated annual numbers of US acute-care hospital patients at risk for venous thromboembolism. Am J Hematol 2007;82:777-782.

52. Di Minno MN, Ambrosino P, Ambrosini F, Tremoli E, Di Minno G, Dentali F. Prevalence of deep vein thrombosis and pulmonary embolism in patients with superficial vein thrombosis: a systematic review and meta-analysis. J Thromb Haemost 2016;14:964-972.

53. Moheimani F, Jackson DE. Venous thromboembolism: classification, risk factors, diagnosis, and management. ISRN Hematol 2011;2011:124610.

54. Silverstein MD, Heit JA, Mohr DN, Petterson TM, O`Fallon WM, Melton LJ 3rd. Trends in the incidence of deep vein thrombosis and pulmonary embolism: a 25 -year population-based study. Arch Intern Med 1998;158:585-593.

55. Naess IA, Christiansen SC, Romundstad P, Cannegieter SC, Rosendaal FR, Hammerstrøm J. Incidence and mortality of venous thrombosis: a population-based study. J Thromb Haemost 2007;5:692-699.

56. Heit JA, Kobbervig CE, James AH, Petterson TM, Bailey KR, Melton LJ 3rd. Trends in the incidence of venous thromboembolism during pregnancy or postpartum: a 30-year population-based study. Ann Intern Med 2005;143:697-706.

57. Virkus RA, Løkkegaard EC, Bergholt T, Mogensen U, LanghoffRoos J, Lidegaard $\varnothing$. Venous thromboembolism in pregnant and puerperal women in Denmark 1995-2005. A national cohort study. Thromb Haemost 2011;106:304-309.

58. Lobastov K, Barinov V, Schastlivtsev I, Laberko L, Rodoman G, Boyarintsev V. Validation of the Caprini risk assessment model for venous thromboembolism in high-risk surgical patients in the background of standard prophylaxis. J Vasc Surg Venous Lymphat Disord 2016;4:153-160.

59. Nguyen GC, Bernstein CN, Bitton A, et al. Consensus statements on the risk, prevention, and treatment of venous thromboembolism in inflammatory bowel disease: Canadian Association of Gastroenterology. Gastroenterology 2014;146:835-848.
60. Kaddourah O, Numan L, Jeepalyam S, Abughanimeh O, Ghanimeh MA, Abuamr K. Venous thromboembolism prophylaxis in inflammatory bowel disease flare-ups. Ann Gastroenterol 2019;32:578-583.

61. Dwyer JP, Javed A, Hair CS, Moore GT. Venous thromboembolism and underutilisation of anticoagulant thromboprophylaxis in hospitalised patients with inflammatory bowel disease. Intern Med J 2014;44:779-784.

62. Papa A, Gerardi V, Marzo M, Felice C, Rapaccini GL, Gasbarrini A. Venous thromboembolism in patients with inflammatory bowel disease: focus on prevention and treatment. World J Gastroenterol 2014;20:3173-3179.

63. Tinsley A, Naymagon S, Trindade AJ, Sachar DB, Sands BE, Ullman TA. A survey of current practice of venous thromboembolism prophylaxis in hospitalized inflammatory bowel disease patients in the United States. J Clin Gastroenterol 2013;47:e1-e6.

64. van Zaane B, Nur E, Squizzato A, et al. Systematic review on the effect of glucocorticoid use on procoagulant, anti-coagulant and fibrinolytic factors. J Thromb Haemost 2010;8:2483-2493.

65. Johannesdottir SA, Horváth-Puhó E, Dekkers OM, et al. Use of glucocorticoids and risk of venous thromboembolism: a nationwide population-based case-control study. JAMA Intern Med 2013;173:743-752.

66. Boeltz S, Amini P, Anders HJ, et al. To NET or not to NET: current opinions and state of the science regarding the formation of neutrophil extracellular traps. Cell Death Differ 2019;26:395-408.

67. Chrysanthopoulou A, Kambas K, Stakos D, et al. Interferon lambda1/ IL-29 and inorganic polyphosphate are novel regulators of neutrophildriven thromboinflammation. J Pathol 2017;243:111-122.

68. Kambas K, Chrysanthopoulou A, Vassilopoulos D, et al. Tissue factor expression in neutrophil extracellular traps and neutrophil derived microparticles in antineutrophil cytoplasmic antibody associated vasculitis may promote thromboinflammation and the thrombophilic state associated with the disease. Ann Rheum Dis 2014;73:1854-1863.

69. Papay P, Miehsler W, Tilg H, et al. Clinical presentation of venous thromboembolism in inflammatory bowel disease. J Crohns Colitis 2013;7:723-729.

70. Angelidou I, Chrysanthopoulou A, Mitsios A, et al. REDD1/ autophagy pathway is associated with neutrophil-driven IL-1 $\beta$ inflammatory response in active ulcerative colitis. J Immunol 2018;200:3950-3961.

71. Heits F, Stahl M, Ludwig D, Stange EF, Jelkmann W. Elevated serum thrombopoietin and interleukin-6 concentrations in thrombocytosis associated with inflammatory bowel disease. J Interferon Cytokine Res 1999;19:757-760.

72. Harries AD, Fitzsimons E, Fifield R, Dew MJ, Rhoades J. Platelet count: a simple measure of activity in Crohn's disease. $\mathrm{Br}$ Med $J$ (Clin Res Ed) 1983;286:1476.

73. Nielsen OH, Vainer B, Madsen SM, Seidelin JB, Heegaard NH. Established and emerging biological activity markers of inflammatory bowel disease. Am J Gastroenterol 2000;95:359-367.

74. Kambas K, Markiewski MM, Pneumatikos IA, et al. C5a and TNF-alpha up-regulate the expression of tissue factor in intraalveolar neutrophils of patients with the acute respiratory distress syndrome. J Immunol 2008;180:7368-7375.

75. Ritis K, Doumas M, Mastellos D, et al. A novel C5a receptortissue factor cross-talk in neutrophils links innate immunity to coagulation pathways. J Immunol 2006;177:4794-4802.

76. Walsh PN. Platelet coagulation-protein interactions. Semin Thromb Hemost 2004;30:461-471.

77. Hammer GP, du Prel JB, Blettner M. Avoiding bias in observational studies: part 8 in a series of articles on evaluation of scientific publications. Dtsch Arztebl Int 2009;106:664-668.

78. Stein PD, Henry JW. Prevalence of acute pulmonary embolism among patients in a general hospital and at autopsy. Chest 1995;108:978-981. 


\section{Supplementary material}

\section{Search Strategy}

Text words and, if applicable, database subject heading fields used to perform a systematic literature search in PubMed, Scopus and Cochrane library.

\section{PubMed}

Research Question: What is the risk of venous thromboembolic events in patients with inflammatory bowel disease? Filters applied: English language, Humans

Concept 1: Venous thromboembolism

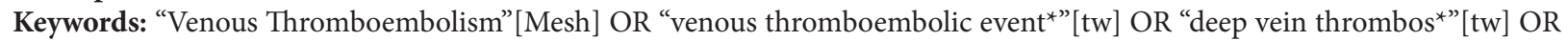
"pulmonary embolism" [tw] OR vte OR pe OR dvt

Mesh: "Venous Thromboembolism"[Mesh]

Concept 2: Inflammatory bowel disease

Keywords: "Inflammatory Bowel Diseases"[Mesh] OR “inflammatory bowel disease*”[tw] OR "ulcerative colitis" [tw] OR "crohn's disease" [tw] OR ibd

Mesh: "Inflammatory Bowel Diseases"[Mesh]

("Venous Thromboembolism" [MeSH Terms] OR "venous thromboembolic event" [Text Word] OR "deep vein thrombos"”[Text Word] OR "pulmonary embolism”[Text Word] OR vte OR pe OR dvt) AND (“Inflammatory Bowel Diseases”[MeSH Terms] OR “inflammatory bowel disease ${ }^{\star}$ "[Text Word] OR “ulcerative colitis”[Text Word] OR “crohn's disease"[Text Word] OR ibd) AND ((humans[Filter]) AND (english[Filter]))

\section{Results: 419}

\section{Scopus}

Research Question: What is the risk of venous thromboembolic events in patients with inflammatory bowel disease? Filters applied: English Language

Concept 1: Inflammatory bowel disease

Keywords: Concept 2: Venous thromboembolism

Keywords: "venous thromboembolism" OR “deep vein thrombosis" OR "pulmonary embolism” OR dvt OR pe OR vte

Keywords: "inflammatory bowel disease" OR "ulcerative colitis" OR "crohn's disease" OR ibd

TITLE-ABS-KEY ("inflammatory bowel disease" ("venous thromboembolism" OR "deep vein thrombosis" OR "pulmonary embolism" OR dvt OR pe OR vte ) AND ( LIMIT-TO ( LANGUAGE , “English”))

\section{Results: 1068}

\section{Cochrane Library}

\#1 (“inflammatory bowel disease”):ti,ab,kw OR (“Crohn’s disease”):ti,ab,kw OR (“IBD”):ti,ab,kw OR (“ulcerative colitis”):ti,ab,kw \#2 (“venous thromboembolism”):ti,ab,kw OR (“pulmonary embolism”):ti,ab,kw OR (“deep vein thrombosis”):ti,ab,kw OR (vte):ti,ab,kw OR (dvt):ti,ab,kw OR (pe):ti,ab,kw

\#3 \#1 and \#2

\section{Results: 60}

